\title{
NG-TRAP: Measuring neutron capture cross-sections of short-lived fission fragments
}

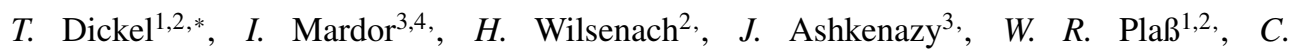
Scheidenberger ${ }^{1,2,5}$, and M. I. Yavor ${ }^{6,}$

${ }^{1}$ GSI Helmholtzzentrum für Schwerionenforschung GmbH, 64291 Darmstadt, Germany

${ }^{2}$ Justus-Liebig-Universität Gießen, 35392 Gießen, Germany

${ }^{3}$ Soreq Nuclear Research Center, Yavne 81800, Israel

${ }^{4}$ Tel Aviv University, Tel Aviv 69978, Israel

${ }^{5}$ Helmholtz Forschungsakademie Hessen für FAIR (HFHF), GSI Helmholtzzentrum für Schwerionenforschung, Campus Gießen, 35392 Gießen, Germany

${ }^{6}$ Institute for Analytical Instrumentation of the Russian Academy of Sciences, 190103 St. Petersburg, Russia

\begin{abstract}
We lack significant nuclear physics input to understand the rapid-neutron capture (r-)process fully. The r-process is the source of half the elements heavier than iron and the only way to produce the long-lived actinides we find on earth. This process's key nuclear physics inputs are nuclear masses, cross-sections of $(\mathrm{n}, \gamma)$ and $(\gamma, \mathrm{n})$, and decay half-lives and branching ratios of neutron-rich isotopes. However, there is currently no method to directly measure neutron-induced reaction rates on short-lived nuclides, so there is no experimental data for the primary nuclear reaction that drives the r-process. We show here a conceptual design of a novel approach to access this information experimentally. The idea is to form a target of short-lived isotopes by confining them as ions in a radio-frequency (RF) trap. Next, they are irradiated with an intense neutron flux, and the reaction products are identified by mass spectrometry. The chosen method is a two-stage process in the presence of high neutron fluxes. The first process is neutron-induced fission in a thin actinide foil to create fission fragments. These fragments are slowed down in a cryogenic stopping cell before being filtered through a radio frequency quadrupole (RFQ) system. The RFQ system selects fission fragments of a specific atomic mass number $A$ and confines them to a small volume in an RF trap, where they are irradiated for a second time in a controlled manner. The resultant $A+1$ isotopes are mass-selectively transported to a multiple-reflection time-of-flight mass spectrometer, where the reaction products are identified and counted.
\end{abstract}

\section{Background}

One of the major open questions in physics is the understanding of the abundance distribution of the heaviest elements, and identification of the astrophysical sites of their production

\footnotetext{
*e-mail: t.dickel@gsi.de
} 
[1]. Astrophysical findings, such as the recent spectroscopic identification of r-process nucleosynthesis in a neutron-star merger [2], provide crucial insights into elemental formation within these newly observed phenomena. Complementarily, measured nuclear physics properties, especially for unstable nuclei, are critical input for network calculations that predict abundance distributions and constrain creation sites [3].

It is possible to measure static properties, such as masses, half-lives, energy levels and radii. However, the measurement of dynamic properties, such as neutron induced crosssections (reaction rates), on short-lived nuclei (half-lives shorter than a few days) is currently considered impossible [4]. Even though the main motivation for the measurement of these cross-sections is the nucleosynthesis r-process, some of the data are also important for validating nuclear structure and reaction models far away from the valley of stability, as well as for the safe operation of nuclear power plants and the transmutation of nuclear waste.

Indirect methods for these measurements have been proposed. Neutron capture crosssections may be somewhat constrained by measuring the nuclear level density and the betastrength function of the resultant nuclei [4]. Presently, the only concepts in the literature for a direct measurement method are combining a storage ring with a nuclear reactor [5], or with an accelerator-based spallation source [6], either which serve as a thermal-neutron "target" that is irradiated by circulating short-lived nuclei. However, technical complexity, safety considerations and high cost make these concepts very challenging.

\section{Preparing a target of short-lived fission fragments}

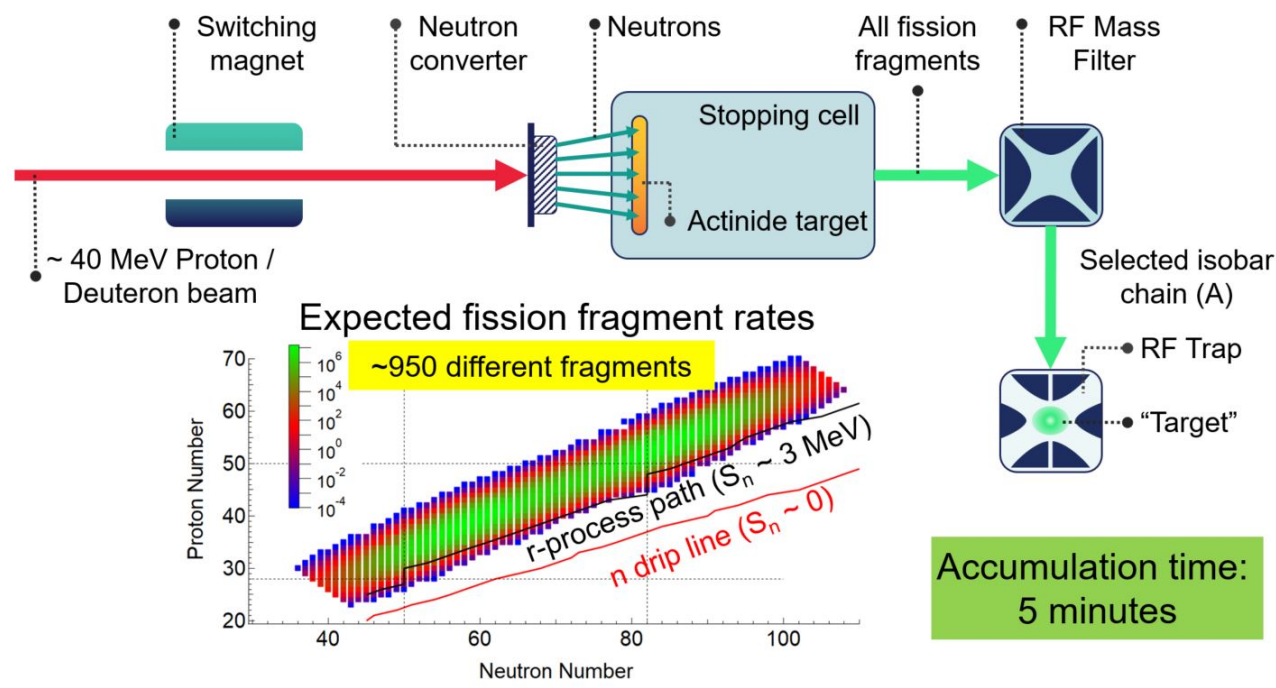

Figure 1. Conceptual layout of the production of short-lived fission fragments, selection of the ions of interest and forming a target of short-lived nuclei in an RF trap. The expected yields (inset) are based on [7], adapted for a gallium-indium neutron converter.

We plan to make use of the intense neutron fluxes that will be available at Soreq Applied Research Accelerator Facility (SARAF-II)[7]. SARAF is a medium-sized high-power accelerator facility that is currently under construction in Soreq Nuclear Research Center at Yavne, Israel, to be completed by 2023. SARAF-II will produce deuteron and proton beams of $40 \mathrm{MeV}$ and $35 \mathrm{MeV}$, respectively, at continuous wave $(\mathrm{CW})$ currents of up to $5 \mathrm{~mA}$. In 
combination with world-exclusive liquid targets, based on the existing liquid-lithium target (LiLiT) that was developed at Soreq [8], the full power of the accelerator will be used to generate high-flux neutron beams at various energy ranges. SARAF-II will include at least two high-flux neutron sources. One source, the liquid gallium-indium beam dump [9], will provide a rate in the range of $10^{15} \mathrm{n} / \mathrm{sec}$ of high energy neutrons, which will generate the needed rate of fission fragments. In a future upgrade, a second source is planned to produce "cold" neutrons (peaked around $5 \mathrm{meV}$ ), with expected fluxes of $10^{10} \mathrm{n} / \mathrm{cm}^{2} / \mathrm{sec}$, which will be used to irradiate the fission fragments and produce a viable measurement rate. The design of the fission fragments production setup has already begun.

We propose to irradiate a thin actinide target $\left({ }^{232} \mathrm{Th}\right.$ or $\left.{ }^{238} \mathrm{U}\right)$ by the high-energy neutron flux, generating $\sim 10^{10}$ fissions/s, see Fig. 1. The fission fragments are released from the thin target undergo thermalization in a cryogenic gas-filled stopping cell (CSC). They are subsequently extracted from the CSC using an RF carpet. Such a CSC has already been developed [10] by the Justus-Liebig University (JLU) Gießen, Germany, and major concepts have been tested at GSI, Darmstadt, Germany [11-13]. The fission fragments are then passed through a RF mass filter (selecting the mass number of interest) to an RF trap, where a selected isobar chain will be stored to form a "target". Efficiencies for escape from the target, thermalization in ionic state in helium, extraction from the high density gas, mass number selection and accumulation in an RF trap are based on experience with similar running experiments $[11,14,15]$. From this we expect a total efficiency for the production, separation and accumulation of approximately $8 \%$. If we assume an isotope at the fission peak with an independent fission yield of $\sim 3 \%$, we get a rate of $\sim 6 \times 10^{7}$ target-nuclei/sec, see inset in Fig. 1. Assuming accumulation of 5 minutes (which enables probing cross-sections down to this half-life range) we can obtain a target of $\sim 2 \times 10^{10}$ ions. We will store ions of all isobars of the selected mass number in the trap and measure their neutron capture cross-sections in parallel. The pseudo potential of the trap needs to be $\sim 500 \mathrm{eV}$, to be able to recapture ions after they undergo beta decay and neutron capture. Thus, our goal is to store about $2 \times 10^{10}$ ions at the same time in the RF trap, for the duration of several minuets. The developments (test setup that includes ion optical elements and electronics) for this are currently ongoing at Tel Aviv University, Israel and the Justus-Liebig University Giessen, Germany.

\section{Neutron capture reaction and detection}

The "target", described in the previous section, will be irradiated by neutrons of a controlled energy spectrum. Following a neutron capture reaction, the resulting nucleus will have recoil energy of about $200 \mathrm{eV}$, so it will be contained in the trap by the pseudo potential and be thermalized by buffer gas cooling. We now need to select the recoil ions from the large amount of unreacted ones without "destroying" the target. This we do by lowering the voltage on one side of the linear RF trap until the first ions spill out of the trap. The ions, at a rate of $1 \times 10^{9} \mathrm{ions} / \mathrm{s}$, will be pushed out of the trap randomly by their thermal motion and spacecharge effects, and consequently be cooled in a short RFQ and then accelerated to a kinetic energy of about $1 \mathrm{keV}$. They are then mass-analyzed in a dipole magnet with a mass resolving power of about 500. This is sufficient to spatially separate recoil $(A+1)$ and unreacted $(A)$ ions. The unreacted ions are guided by electrostatic deflectors back to the linear RF trap (target). The ions spend merely a few milliseconds out of the target, and are then recaptured with high efficiency, as we have demonstrated for a much more challenging case [16], so the number of ions in the target is not depleted significantly.

The reacted ions are guided to the trap of a multiple-reflection time-of-flight mass spectrometer (MR-TOF-MS), which is used for their identification and counting. The MR-TOFMS can handle more than $10^{6}$ ions per second [17], so the dipole magnet only needs to 


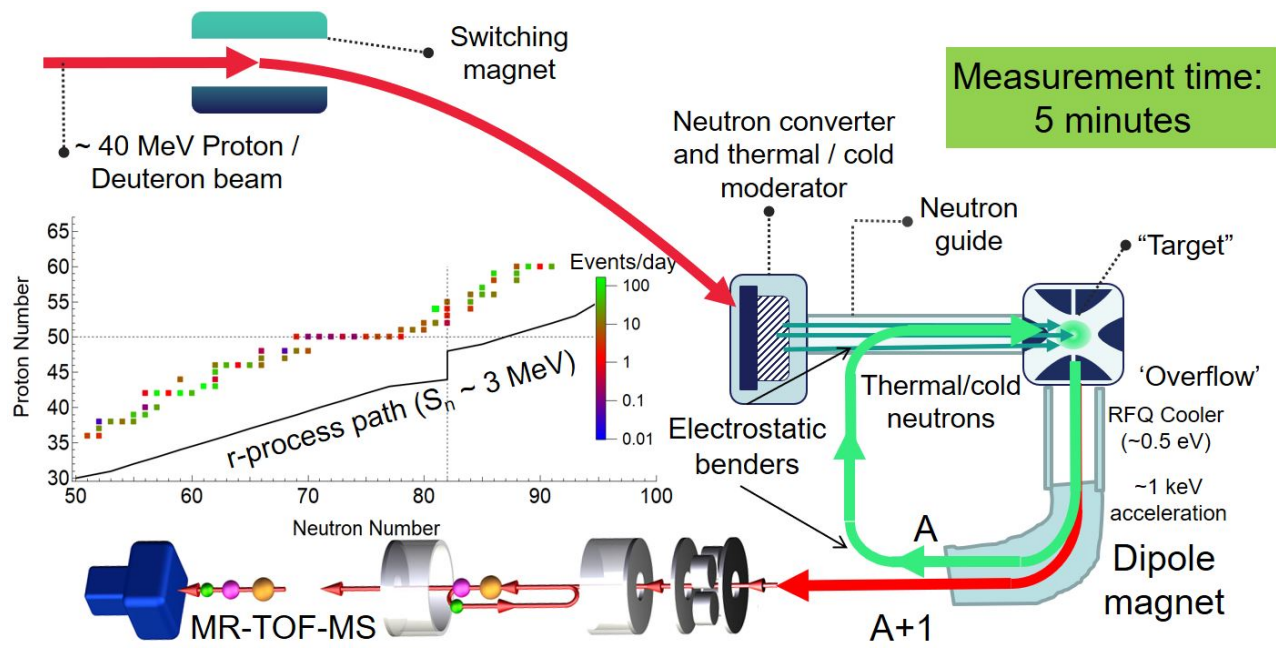

Figure 2. Conceptual layout of the irradiation with cold neutron and detection of reaction products. The inset shows the expected detected event rate per day.

suppress the unreacted ions by 3 orders of magnitude. The counted ions, normalised to the total amount of target nuclei and neutron flux, will infer the desired cross-section. The entire process is depicted in Figure 2. Note that we will measure reaction rates for several nuclei of the selected isobaric-chain simultaneously, which will save on beam time and help to increase statistics. This is possible due to the high mass resolution that can be achieved in modern time-of-flight mass spectrometers $[18,19]$. The typical analysis time will be one minute, which is dominated by the average time it takes the ions to leave the target RF trap, much longer than the detection and identification by the MR-TOF-MS, which takes about 10 ms.

In order to measure neutron capture cross-sections of the fission fragments, we intend to irradiate the trapped ions with cold neutrons. The neutron capture cross-section energy $(E)$ dependence is proportional to $\sim 1 / E^{1 / 2}$ for all isotopes (with resonances in the epithermal region, and rarely also in the thermal region) [20]. In order to experimentally constrain the cross-section values at the region of interest for the r-process (10's to 100 's of $\mathrm{keV}$ ), it is sufficient to provide experimental points at the cold or thermal region, preferably at both [21]. Measurements at both points confirm the $\sim 1 / E^{1 / 2}$ trend, whereas a deviation point to a resonance in this region.

Using cold neutrons has two major advantages. First, because of the $1 / E^{1 / 2}$ behaviour, the cross-sections in this energy region are very high ( $\sim 1$ barn and higher, in some cases by orders of magnitude [22]). Second, cold neutrons can be steered and focused into the target without losses along paths of tens of meters, via usage of so-called "neutron guides". Such guides are available commercially from several companies [23-25] and are used in reactor- and accelerator-based neutron sources (existing and planned [26]). If we assume a cold neutron flux of $10^{10} \mathrm{n} / \mathrm{cm}^{2} / \mathrm{sec}$ and a combined ejection and mass measurement efficiency of $50 \%$, we get an overall count rate of a few events per day per barn for neutron capture events. The expected yields for the accessible fission fragments can be found in the inset in Fig. 2 . Since this measurement method is accurate and virtually background-free, we will be able 
to measure cold and/or thermal neutron capture cross-sections of numerous unstable fission fragments with sufficient statistics within a few days.

It is well known that several nuclei have surprisingly high thermal (and thus also cold) neutron capture cross-sections. Veteran examples include stable ${ }^{157} \mathrm{Gd}\left(2.1 \times 10^{5}\right.$ barn $)$ and the famous "reactor poison" ${ }^{135} \mathrm{Xe}\left(2.6 \times 10^{6} \mathrm{barn}\right)$ with $t_{1 / 2}=9.14 \mathrm{hr}$, and many isotopes with cross-sections in the $10^{2}, 10^{3}$ and even $10^{4}$ barn range. There is currently no nuclear model to predict which isotopes will exhibit such a high resonance in the thermal region. Even recently, another surprisingly high cross-section was published, following the first thermal $(\mathrm{n}, \gamma)$ measurement of ${ }^{88} \mathrm{Zr}\left(t_{1 / 2}=83.4\right.$ days $)$, and the result was $8.6 \times 10^{5}$ barn [27], versus the theoretically predicted value of $\sim 10$ barns [22]. We thus expect that in our systematic survey of cold and thermal neutron capture cross-sections of fission fragments, we will also discover new "surprises", which may promote theoretical explanations for this phenomenon. Outliers in the range of $10^{2}$ and $10^{3}$ barn will be accessible with our method within a few hours of measurement.

\section{Summary and Outlook}

We have shown the conceptual design of a novel method to measure neutron capture crosssection of short-lived fission fragments, down to half-lives in the scale of minutes. This is a paradigm change as it is deemed so far as technically impossible to perform such measurements. The measurements will have a significant impact, and will provide invaluable input to astrophysical nucleosynthesis, nuclear structure and reaction models, and nuclear reactor safety and nuclear waste transmutation. Confining short-lived isotopes as ions in a RF trap could also be applied to $(\gamma, \mathrm{n}),(\mathrm{n}, 2 \mathrm{n})$ and $(\alpha, \mathrm{n})$, in addition to $(\mathrm{n}, \gamma)$. Installing a similar system at Extreme Light Infrastructure - Nuclear Physics (ELI-NP) [28] with its unprecedented brilliant gamma beams could allow to directly probe the r-process relevant $(\gamma, \mathrm{n})$ cross-sections.

\section{Acknowledgements}

This work was supported by the German Research Foundation (DFG) Grant No. 57564554, by Justus-Liebig-Universität Gießen and GSI under the JLU-GSI strategic Helmholtz partnership agreement, and by the Israel Ministry of Energy, Research Grant No. 220-11-052.

\section{References}

[1] A. Arcones, G. Martínez-Pinedo, Phys. Rev. C 83, 045809 (2011)

[2] E. Pian, P. D’Avanzo, S. Benetti, M. Branchesi, E. Brocato, S. Campana, E. Cappellaro, S. Covino, V. D’Elia, J.P.U. Fynbo et al., Nature 551, 67 (2017)

[3] M. Mumpower, R. Surman, G. McLaughlin, A. Aprahamian, Progress in Particle and Nuclear Physics 86, 86 (2016)

[4] A. Larsen, A. Spyrou, S. Liddick, M. Guttormsen, Progress in Particle and Nuclear Physics 107, 69 (2019)

[5] R. Reifarth, Y.A. Litvinov, Phys. Rev. ST Accel. Beams 17, 014701 (2014)

[6] R. Reifarth, K. Göbel, T. Heftrich, M. Weigand, B. Jurado, F. Käppeler, Y.A. Litvinov, Phys. Rev. Accel. Beams 20, 044701 (2017)

[7] I. Mardor, O. Aviv, M. Avrigeanu, D. Berkovits, A. Dahan, T. Dickel, I. Eliyahu, M. Gai, I. Gavish-Segev, S. Halfon et al., The European Physical Journal A 54, 91 (2018)

[8] S. Halfon, A. Arenshtam, D. Kijel, M. Paul, L. Weissman, O. Aviv, D. Berkovits, O. Dudovitch, Y. Eisen, I. Eliyahu et al., Review of Scientific Instruments 85, 056105 (2014) 
[9] I. Eliyahu, S. Vaintraub, I. Mardor, A. Arenshtam, E. Shvero, E. Reinfeld, O. Ozery, M. Alpha, R. Raz, M. Bukai et al., High Power Beam Dump and Neutron Sources for SARAF Phase II, http://www-llb.cea.fr/UCANS8-2019/Eliyahu_poster.pdf/, accessed: 2021-11-25

[10] T. Dickel, W.R. Plaß, H. Geissel, F. Heiße, I. Miskun, S. Purushothman, M.P. Reiter, A.K. Rink, C. Scheidenberger, NIM B 376, 216 (2016)

[11] W.R. Plaß, T. Dickel, S. Purushothaman, P. Dendooven, H. Geissel, J. Ebert, E. Haettner, C. Jesch, M. Ranjan, M.P. Reiter et al., NIM B 317, 457 (2013)

[12] M. Ranjan, P. Dendooven, S. Purushothaman, T. Dickel, M. Reiter, S. Ayet San Andrés, E. Haettner, I. Moore, N. Kalantar-Nayestanaki, H. Geissel et al., NIM A 770, 87 (2015)

[13] Mardor, Israel, Dickel, Timo, Amanbayev, Daler, Ayet San Andrés, Samuel, Beck, Sönke, Benyamin, David, Bergmann, Julian, Constantin, Paul, Cléroux Cuillerier, Alexandre, Geissel, Hans et al., EPJ Web Conf. 239, 02004 (2020)

[14] W.R. Plaß, T. Dickel, I. Mardor, S. Pietri, H. Geissel, C. Scheidenberger, D. Amanbayev, S. Ayet San Andrés, J. Aysto, D. Balabanski et al., Hyperfine Interactions 240, 73 (2019)

[15] E. Haettner, W.R. Plaß, U. Czok, T. Dickel, H. Geissel, W. Kinsel, M. Petrick, T. Schäfer, C. Scheidenberger, NIM A 880 (2018)

[16] T. Dickel, W.R. Plaß, W. Lippert, J. Lang, M.I. Yavor, H. Geissel, C. Scheidenberger, J. Am. Soc. Mass Spectr. 28, 1079 (2017)

[17] T. Dickel, W.R. Plaß, A. Becker, U. Czok, H. Geissel, E. Haettner, C. Jesch, W. Kinsel, M. Petrick, C. Scheidenberger et al., NIM A 777, 172 (2015)

[18] S. Ayet San Andrés, C. Hornung, J. Ebert, W.R. Plaß, T. Dickel, H. Geissel, C. Scheidenberger, J. Bergmann, F. Greiner, E. Haettner et al., Phys. Rev. C 99, 064313 (2019)

[19] I. Mardor, S. Ayet San Andrés, T. Dickel, D. Amanbayev, S. Beck, J. Bergmann, H. Geissel, L. Gröf, E. Haettner, C. Hornung et al., Phys. Rev. C 103, 034319 (2021)

[20] K.S. Krane, Introductory Nuclear Physics (Wiley India, 2008), ISBN 9788126517855, https://books.google.de/books?id=mkZjC1TLXh8C

[21] R. Reifarth, K. Schwarz, F. Kappeler, The Astrophysical Journal 528, 573 (2000)

[22] A. Koning, D. Rochman, Nuclear Data Sheets 113, 2841 (2012)

[23] Mirrotron, https://mirrotron.com/en, accessed: 2019-08-30

[24] Swiss Neutronics, https://www. swissneutronics.ch/, accessed: 2019-08-30

[25] Neutron Guide, http: //www . neutronguide.com/, accessed: 2019-08-30

[26] K.H. Andersen, M. Bertelsen, L. Zanini, E.B. Klinkby, T. Schönfeldt, P.M. Bentley, J. Saroun, Journal of Applied Crystallography 51, 264 (2018)

[27] J.A. Shusterman, N.D. Scielzo, K.J. Thomas, E.B. Norman, S.E. Lapi, C.S. Loveless, N.J. Peters, J.D. Robertson, D.A. Shaughnessy, A.P. Tonchev, Nature 565, 328 (2019)

[28] N. Zamfir, The European Physical Journal Special Topics 223, 1221 (2014) 